\title{
Energy Distribution and Exchange Between Spatial Harmonics in Bending Wave Phononic Crystals
}

\author{
Bart Van Damme* and Armin Zemp \\ Empa, Laboratory for Acoustics/Noise Control, CH-8600 Dübendorf, Switzerland
}

(Received 8 March 2018; revised manuscript received 18 May 2018; published 3 July 2018)

\begin{abstract}
Resonant metamaterials and phononic crystals are widely studied, with the aim of controlling and altering elastic wave propagation in solids. Beams with varying stiffness along the length are an archetypal example of a phononic crystal for bending waves, since the resulting nontrivial dispersion relation can be modeled analytically. In this work, the complex dispersion curve over the complete audible frequency range is measured using the inhomogeneous wave-correlation (IWC) technique, and compared to a unitcell model and an explicit model of the finite beam. Multiple band gaps, each with a different attenuation coefficient, are predicted and validated. An iterative implementation of IWC allows the identification of a spatial spectrum of wave numbers, responsible for the intricate beam displacement shape. The energy carried by the fundamental wave number and its Bloch (sub)harmonics are determined, showing that the main dispersion branch is monotonically increasing. It is shown that only a small fraction of the wave energy travels along branches with a negative slope in the first Brillouin zone. Additionally, in band gaps affecting only bending waves, energy is converted to other wave types, such as longitudinal waves. This approach offers some important insights into the physics of the wave propagation, which cannot be gathered from unit-cell modeling alone.
\end{abstract}

DOI: 10.1103/PhysRevApplied.10.014001

\section{INTRODUCTION}

It is well known that elastic structures can be designed to tune wave propagation at will [1,2]. Applications are, among others, wave focusing [3], wave guiding [4,5], acoustic cloaking [6], wave-type conversion [7], and the creation of frequency bands with high attenuation, the socalled band gaps [8-12]. The latter can be obtained for acoustic waves in air, but also for longitudinal, shear, surface, and flexural elastic waves. Such structures can be classified into two families: phononic crystals and resonant metamaterials. In the former case, band gaps occur when waves are scattered by a periodic structure, and then undergo destructive interference. This requires that the distance between two scatterers be of the order of a wavelength [13]. Moreover, the frequency bands with high attenuation depend in general on the propagation direction of the incoming wave. The latter is an increasingly popular approach, making use of resonators connected to the structure. The resonators are tuned to absorb the frequencies of interest, and separated by distances much smaller than a wavelength. These resonators can be mechanical [14] or electromagnetic [15].

Ever since Mead's general paper on the modeling of elastic waves in periodic structures [16], dispersion

\footnotetext{
*bart.vandamme@empa.ch
}

calculations have been mostly limited to the first Brillouin zone (BZ), in other words for wavelengths longer than one period of the structure. The application of periodic boundaries in the wave number domain on a geometric unit cell significantly reduces the calculation effort, especially when the finite-element method is used [17]. Because of this convenience, resonators in metamaterials are often placed periodically, although this is not strictly necessary for wave attenuation [18]. For two- and three-dimensional (2D and $3 \mathrm{D})$ periodic structures, the symmetry of the unit cell can be taken into account to reduce the information contained in the first BZ to the one of a contour. The dispersion along the edge of the irreducible BZ contains all relevant wavepropagation information for an infinite assembly of unit cells [19].

Beyond the wave numbers in the first BZ, higher values can be considered: The real part of the wave number exhibits periodicity $2 \pi / L$, where $L$ is the size of the unit cell in a given direction. The higher wave numbers in this infinite series of solutions are called Bloch harmonics, and their existence has been shown experimentally both in photonic crystals [20] and for ultrasound waves in phononic crystals [21]. Multiple wave types (bending, longitudinal, torsional) make the full dispersion relation even more intricate. For a single-frequency harmonic excitation, an infinite number of wave numbers can be excited. Unitcell analysis does not allow for the prediction of the energy 

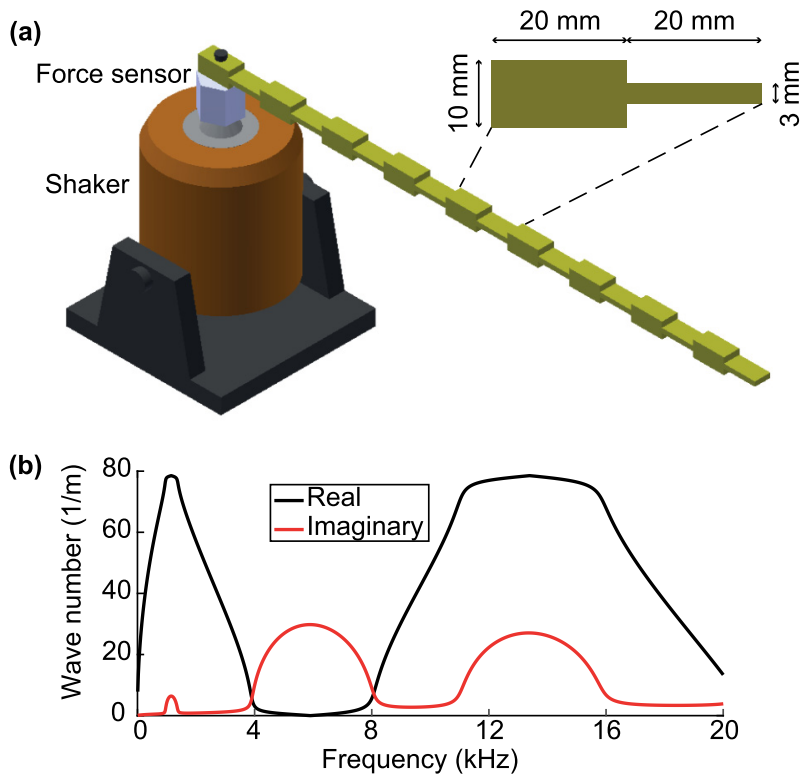

FIG. 1. (a) Experimental setup of a beam with periodic thickness variation and (b) analytical dispersion curve for bending waves in the first BZ.

distribution between the wave modes and their Bloch harmonics, since it is determined by the size of the sample and the excitation source. The energy participation of each mode can be calculated using, e.g., the modal participation factor [22], or using wave-mode analysis [23]. To address these aspects of the dynamic behavior, a finite assembly and the exact excitation mechanism have to be modeled explicitly. This can, for instance, be done using finite differences or finite-element models. Although feasible for samples of moderate size, this approach is impractical for large 2D and 3D structures with intricate geometries, since it leads to excessive calculation times.

The location of band gaps is predicted correctly using a unit-cell model, but this gives no information on which dispersion branches are most responsible for a structure's deformation and therefore have to be attenuated. More advanced applications such as lensing or cloaking depend on knowledge of the phase velocity of waves, which depends on the BZ in which most of the energy travels. It is therefore of great importance to take the wave-energy distribution into account.

In this paper, we show some repercussions of unit-cell analysis by considering the well-known example of beams with a periodically varying thickness, depicted schematically in Fig. 1(a). Beams with periodic cross sections, built out of alternating materials, or with periodic elastic supports, have been thoroughly investigated analytically [24-27]. Such phononic crystals exhibit multiple wide band gaps, and dispersion branches with a negative slope in the first BZ. In this work, the wave dispersion and the harmonic deflection of a finite beam are calculated using both unit-cell analysis and full-beam modeling. Material damping and shear effects are taken into account, in order to produce realistic results over the entire audible frequency range (50-19000 Hz). In a second step, the analytically calculated results are validated by laser vibrometer measurements carried out on a 3D-printed polyamide sample. Finally, an iterative expansion of the inhomogeneous wave-correlation (IWC) method is proposed in order to extract a series of spatial harmonics and their respective amplitudes, which gives us the possibility of addressing the mentioned limitations of unit-cell modeling: determining the main contributing wave number at a given excitation.

\section{METHODS}

\section{A. Analytical model and the dispersion relation}

The deflection and dispersion of a beam with periodic properties (varying bending stiffness or periodic elastic support) can be calculated using a transfer-matrix formulation [24]. For this work, the thick-beam model was used, since at higher frequencies the shear effects become increasingly important:

$$
\begin{aligned}
& E I \frac{\partial^{4} w}{\partial x^{4}}+\rho A \frac{\partial^{2} w}{\partial t^{2}} \\
& \quad-\left(\rho I+\frac{E I \rho}{K A G}\right) \frac{\partial^{4} w}{\partial x^{2} \partial t^{2}}+\frac{\rho^{2} I}{K G} \frac{\partial^{4} w}{\partial t^{4}}=0 .
\end{aligned}
$$

The material parameters are Young's modulus $E$, the shear modulus $G$, and the mass density $\rho$. The geometry is given by the varying thickness $h$ and width $b$, the beam cross section $A=b h$, and the moment of area $I=b h^{3} / 12$. The constant $K=5 / 6$ captures the through-the-thickness shear-force variation. At each interface between thick and thin sections, the continuity of the displacement, the bending angle, the bending moment, and the shear force are implemented as boundary conditions. The calculation of the deformation of a full beam with ten periods is done using a free boundary at one end and a harmonic force at the other. In each section, four coefficients are to be found, one for each wave term in the general solution $A \sin \left(k_{1} x\right)+$ $B \cos \left(k_{2} x\right)+C \exp \left(k_{3} x\right)+D \exp \left(k_{4} x\right)$. The presence of trigonometric and exponential functions results in a badly conditioned system of 80 linear equations, making the solution tedious for beams with more than ten periods.

The dispersion relation of an infinite periodic beam is calculated using a unit cell with periodic boundary conditions. This results in just eight equations (four at the interface and four periodic boundary conditions), thus avoiding the numerical problems encountered in solving the equations for the entire beam.

The dispersion for lateral bending, in the plane perpendicular to the input force, can be found in a straightforward way by switching the values for the width and the thickness. The results for longitudinal waves are based on the 
wave equation for a motion $u$ along the beam axis:

$$
E \frac{\partial^{2} u}{\partial x^{2}}+\rho \frac{\partial^{2} u}{\partial t^{2}}=0
$$

The boundary conditions at each interface are defined by the continuity of displacement and strain.

\section{B. Experimental setup}

A 10-mm-wide beam with a varying thickness profile (10 $\mathrm{mm}$ and $3 \mathrm{~mm}$ ), each section being $20 \mathrm{~mm}$ long, was 3D-printed in polyamide. The Young's and shear moduli were derived by fitting the bending-wave dispersion of a solid, rectangular beam printed on the same machine, using the method described in Ref. [28]. The measured dispersion relation is fitted to the analytical one by optimizing the material properties, resulting in the values $E=1.9 \mathrm{GPa}$, $G=1.4 \mathrm{GPa}$, and $\rho=1135 \mathrm{~kg} / \mathrm{m}^{3}$. Material damping was introduced using an imaginary value for $E$, equal to $5 \%$ of the real value.

For the measurements, the beam was screwed onto a PCB 208C01 force sensor, which was in turn connected to an LDS V201 permanent magnet shaker [Fig. 1(a)]. The other end of the beam was left free, allowing for the analytical implementation of a free end. The vertical (parallel to the input force) beam-deflection velocity was measured using a Polytec PSV-400 scanning laser Doppler vibrometer (SLDV). The shaker was driven by a sine sweep ranging from 50 to $19000 \mathrm{~Hz}$, generated by the Polytec measurement system. The vibration velocity at 87 points, ordered in a linear grid with a 4-mm spacing, was used, spanning the entire length of the structured beam. For the further data analysis, the $\mathrm{H} 1$ frequency response function (FRF), defined as the ratio of the complex velocity and force spectrum, was calculated.

\section{Iterative inhomogeneous wave correlation}

The IWC method [28-30] delivers the complex dispersion relation based on the knowledge of the FRF along a line. The method compares the measured signal at a chosen frequency, $s\left(x, f_{0}\right)$, to a damped traveling wave $\hat{o}=\exp [i(k+i g) x]$ by calculating the correlation

$$
\Im(k, g)=\frac{\left|\int s\left(x, f_{0}\right) \hat{o}^{*}(x, k, g) d x\right|}{\sqrt{\int\left|s\left(x, f_{0}\right)\right|^{2} d x \int|\hat{o}(x, k, g)|^{2} d x}},
$$

where the integration is performed over the length of the beam. For discrete measurement points, the integral is reduced to a summation. Maximization of the correlation function in terms of $k$ and $g$ (the real and imaginary parts of the wave number) defines the point of best agreement between $s$ and $\hat{o}$. Repetition of the calculation for a series of frequencies returns the full complex dispersion relation. In this work, the IWC method was implemented in MATLAB ${ }^{\circledR}$ The fmincon command was used to find the minimum of $-\Im(k, g)$ within given boundaries.

Multiple less-pronounced secondary maxima are also present in the correlation function. In order to reconstruct the full spatial spectrum of the wave pattern, an iterative method can be applied:

(1) Find the location $\left(k_{1}, g_{1}\right)$ of the absolute maximum using the fmincon algorithm.

(2) Calculate the complex amplitude of this component of the total deflection, by projecting the inhomogeneous wave onto the measured vibration pattern, and normalizing it to the norm of $\hat{o}$ :

$$
A_{1}=\frac{\sum_{i} \hat{o}\left(k_{1}, g_{1}, x_{i}\right) s\left(x_{i}\right)}{\sum_{i} \hat{o}\left(k_{1}, g_{1}, x_{i}\right) \hat{o}^{*}\left(k_{1}, g_{1}, x_{i}\right)} .
$$

(3) Subtract the scaled inhomogeneous wave $A_{1} \hat{o}\left(k_{1}\right.$, $\left.g_{1}, x\right)$ from the measured vibration pattern and go back to step 1.

As long as the signal is not buried under the noise floor, the method can be repeated. In this work, at least 12 wave numbers could be acquired.

\section{RESULTS}

\section{A. Dynamic deformation inside and outside of band gaps}

In the considered frequency range, four pass bands and three band gaps were identified, as shown in Fig. 1(b). The width of these gaps and their attenuation efficiency (given by the imaginary part of the wave number) vary greatly. The use of complex material properties results in a nonzero value of the imaginary wave number, even in pass bands. Moreover, the real part of the wave number no longer remains constant inside a band gap, but shows a smooth transition in accordance with other models [30,31]. This means that there are no discontinuities in the waves' group velocities, as is the case if no material damping is considered.

The measured deflection profile of the full beam is in very good agreement with the calculated deformations, as shown in Fig. 2. It is clear that the beam deflection is more attenuated in the band gap areas (b)-(d) than in the pass bands (a)-(c). The analytical solution also clearly shows that the bending mainly takes place in the thin sections, sometimes leading to sharp angles and sudden changes in the deformation shape. Temporal Fourier analysis of angular signals, such as rectangular or sawtooth waves, reveals the presence of harmonics of the fundamental frequency. In the next section, it is shown that the same principle is valid for spatial deformations. 
(a)

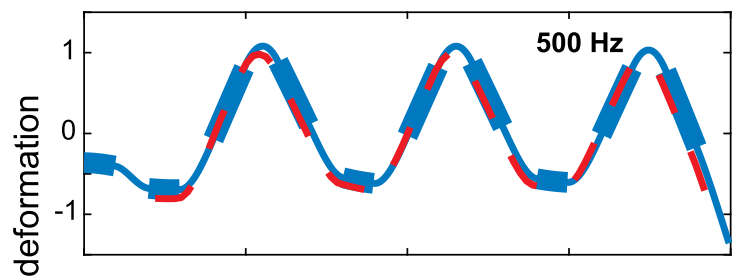

(b)

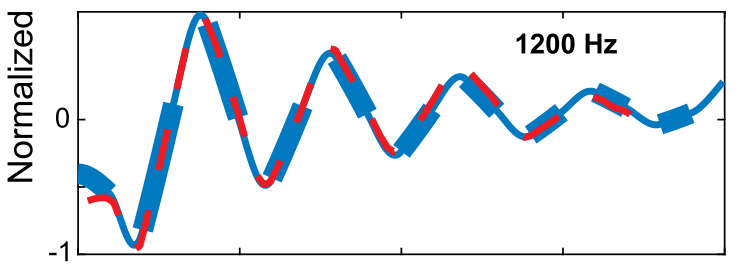

(c)

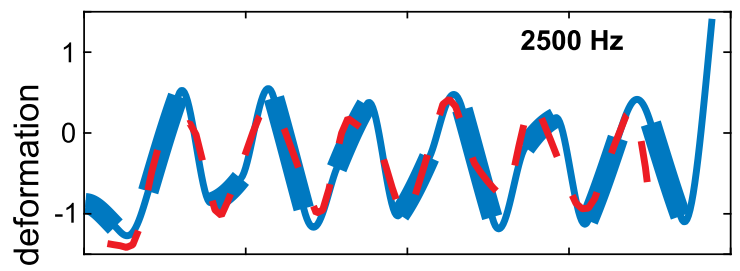

(d)

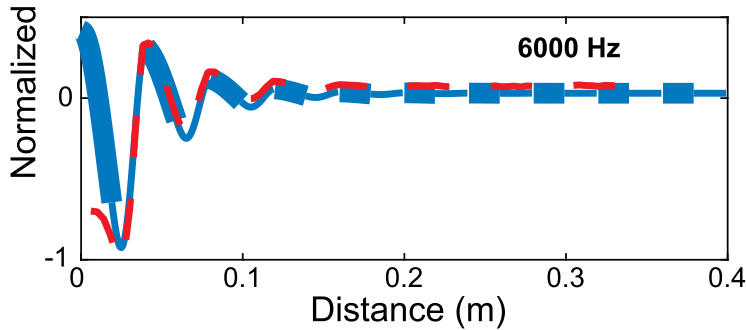

FIG. 2. Displacement of a periodic beam excited by a harmonic force: the analytical calculation of a finite beam (solid line, showing the thickness profile) and measurement (dashed line) for four frequencies. Images (a) and (c) show results in the first two pass bands, while images (b) and (d) are vibration shapes in the first band gaps.

\section{B. Experimental determination of the complex dispersion curve}

From the measured displacement profile, the complex dispersion relation was determined using the IWC method. Figures 3(a) and 3(b) show the real and imaginary parts of the wave number as a function of frequency. The inherent material damping, introduced using an imaginary part of the Young's modulus, results in measurable nonzero values of the imaginary wave number in pass bands and smooth dispersion curves at the edges of the BZ without sudden slope changes. The predicted depth of the band gaps agrees well with the experimental values, although the results become noisy for high attenuation. The main real component of the wave number follows the branch with ever-increasing values, outside the first BZ (BZ0). After each band gap, the wave number containing the highest energy is located in the next BZ (BZ1-BZ2). This agrees with the physical intuition that the wavelength should decrease for increasing frequencies.
However, the intricate deflection profiles shown in Fig. 2 suggest that more than one wavelength is present for each excitation frequency. This is especially true for the sometimes sharp angles at higher frequencies. Subtraction of the main wave component from the full deformation and repetition of the IWC calculation on the remaining signal lead to an iterative scheme that allows a large number of wave numbers to be obtained. The result for the first five iterations is shown in Fig. 3(c). At low frequencies, in the first pass band, only the wave numbers in the first and second BZs emerge. Both right- and left-traveling waves - with positive and negative wave numbers, respectively - are identified. In the second and third pass bands, harmonics up to the third BZ appear. Remarkably, some of the identified wave numbers belong to branches with a negative slope, traditionally called double-negative frequency bands. The phase and group velocities of these waves have opposite signs. The amplitudes of these waves, however, are small compared to the principal waves on branches with a positive slope, as will be shown in the next section.

Within the band-gap areas, highlighted in gray, different wave modes than bending waves were identified. In the second band gap, most of the energy appears as a longitudinal wave in the first and third BZs. The frequency range $12-13 \mathrm{kHz}$ shows the interesting case of a full band gap for the three considered wave modes. In fact, the obtained wave numbers do not agree with any of the predicted dispersion curves. They might, for example, belong to torsional deformation.

\section{Amplitude analysis of Bloch harmonics}

Repetition of the IWC procedure not only allows for the derivation of the wave numbers for higher BZs, but also yields the amplitude of each retrieved mode. Figure 4 shows the result for the first 12 wave modes at six different frequencies. The top row shows frequencies in the first three pass bands. It is clear that most of the wave energy travels as out-of-plane bending waves (solid lines), and that the highest energy is found at ever-increasing wave numbers. A limited amount of energy also travels as lateral bending waves (dash-dotted lines) and longitudinal waves (dashed lines).

The results within band gaps are similar, except that the energy contained in longitudinal and torsional waves is higher compared to the out-of-plane bending. This can be explained by the fact that the bending motion is highly attenuated, whereas the other wave types are not.

For several frequencies, both in pass bands and band gaps, some wave numbers cannot be identified as bending or longitudinal waves. They might be artifacts due to noisy signals when the measured amplitudes are small, which is especially the case in band gaps, or they may denote other wave types, such as torsional waves. 

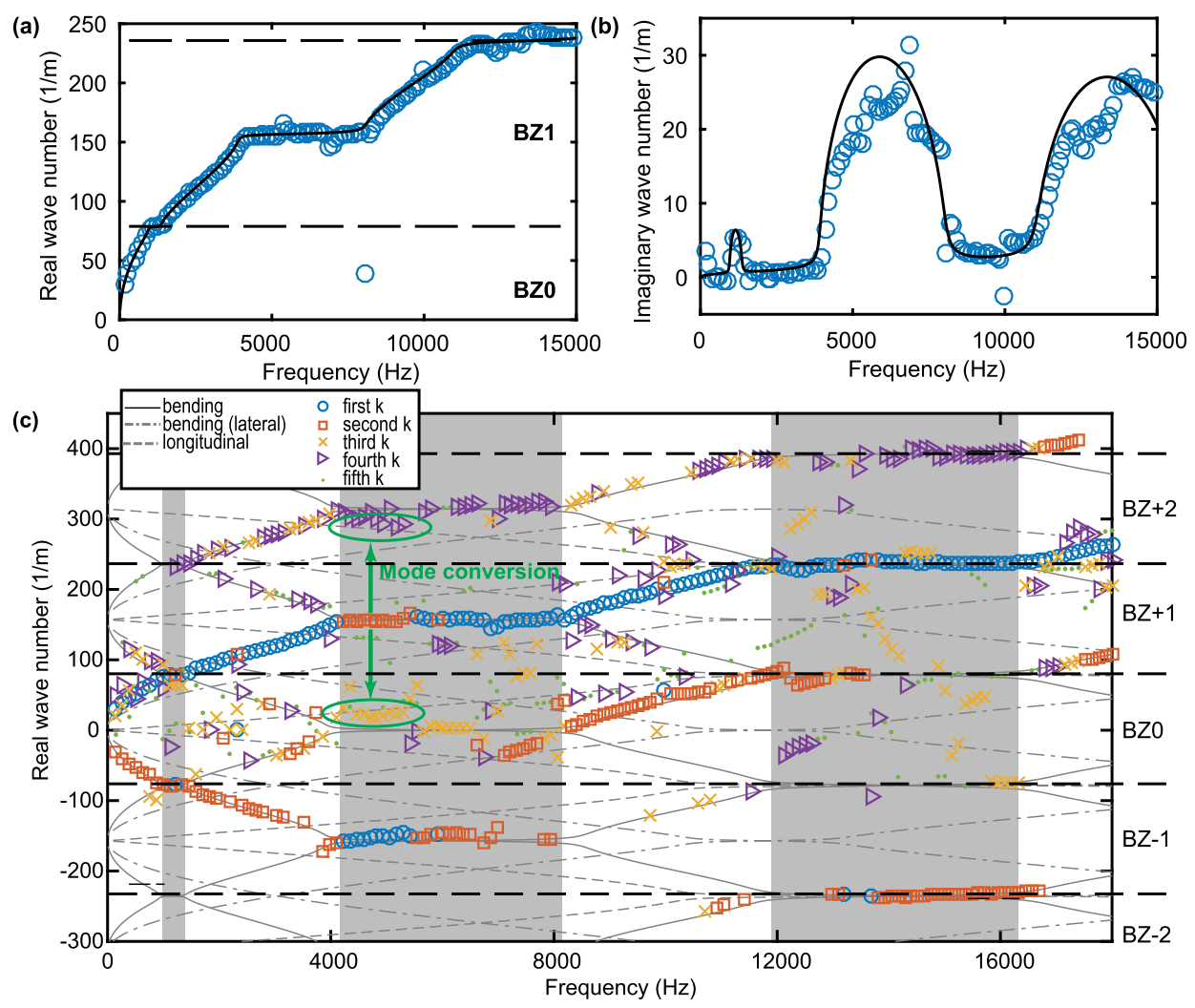

FIG. 3. (a),(b) Experimental complex dispersion curve using the standard IWC method (circles) compared to the analytical calculation (black line). The first BZ is numbered BZ0. The higher BZs, containing Bloch harmonics, are denoted by $\mathrm{BZ} j$, where $j= \pm 1,2$. (c) Experimental result of the iterative IWC method, showing multiple spatial harmonics and the presence of longitudinal beam deformation. The first five wave numbers are shown in order of decreasing amplitude (first to fifth $k$ ). In the second band gap, energy is efficiently converted from bending waves to longitudinal wave modes.

\section{Discussion}

The enhanced iterative IWC method identifies the wavenumber content of harmonic waves in phononic crystals. It shows that a set of waves with varying wavelengths contributes to the total deflection of a phononic crystal when it is excited by a harmonic force. This illustrates the importance of two limitations of modeling periodic media using Floquet boundary conditions. First, when reaching a band gap, the main wave contribution shifts to the nexthigher BZ. Energy travels mainly along monotonically increasing dispersion branches. Second, unit-cell simulations do not give any information about the relative wave amplitudes.

A harmonic excitation results in a series of waves with different wave numbers, following the periodic Bloch spectrum. Experimental and analytical results, compared in Fig. 4, agree very well for frequencies below $10 \mathrm{kHz}$. At higher frequencies, the model and experiment are still similar for the main bending modes, but other wave types are generated as well, and they influence the experiment, as can clearly be seen in Fig. 3(c). In partial bending-wave band gaps, a considerable amount of wave energy is converted to longitudinal waves.

The case of dispersion branches with a negative slope is of particular interest in the literature, since it is a typical example of exotic wave propagation that does not exist in homogeneous elastic materials. Although zones with a negative refraction coefficient are predicted when limiting the analysis to the first BZ, we demonstrate that most of the energy is contained in Bloch harmonics with a positive group velocity. The energy content of wave modes with a negative dispersion slope is typically 50-100 times lower. Although we do not claim that this is a universal result, it highlights the importance of correctly interpreting Floquet analysis limited to the irreducible BZ in continuous media.

\section{CONCLUSIONS}

Elastic waves in beams with a periodic cross section variation exhibit an intricate dispersion relation in the first BZ, resulting in band gaps with varying width and efficiency and negative-slope branches. Results of an analytical unit-cell calculation and a full-beam model are validated experimentally with high accuracy by the inhomogeneous wave-correlation technique, showing that the wave number with the highest contribution is not necessarily located in the first BZ. Taking into account shear effects and material damping results in excellent agreement between the model and experimental values. We developed an iterative version of the IWC method to produce results that cannot be retrieved from unit-cell models: the relative amplitudes of waves in various branches of the dispersion curve, the presence and importance of Bloch harmonics, and the relatively weak contribution of waves with 

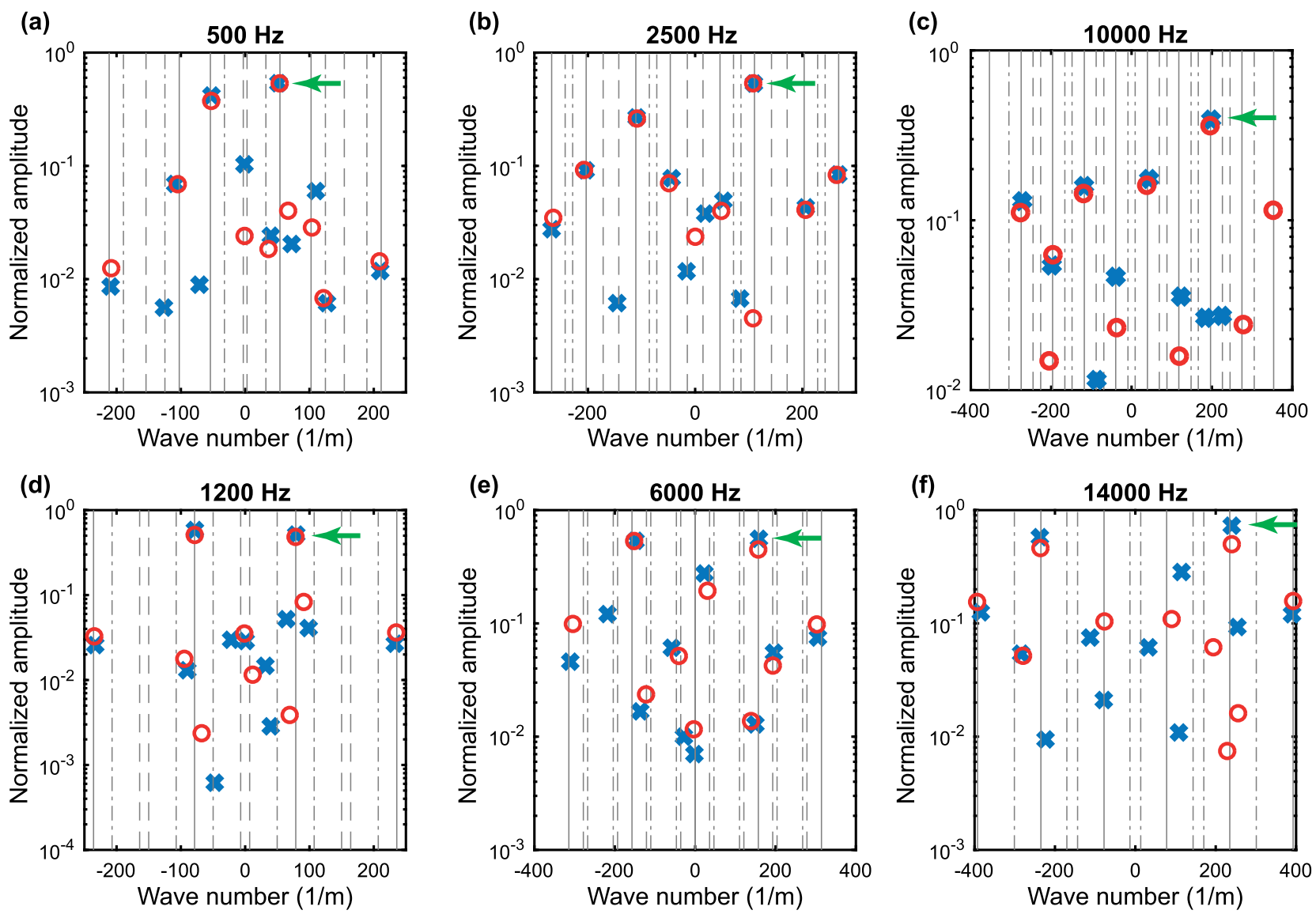

FIG. 4. Amplitudes of the first 12 Bloch harmonics from measurements (crosses) and calculated deflections (circles), retrieved by the iterative IWC method. The mode with the highest amplitude is highlighted by a green arrow. The predicted location of the wave numbers for out-of-plane bending (solid lines), lateral bending (dash-dotted lines), and longitudinal waves (dashed lines) are shown. The calculation was done for six frequencies, three in pass bands (a)-(c) and three in band gaps (d)-(f).

negative-slope dispersion branches. This demonstrates that unit-cell analysis is admittedly a useful tool during the design stage of metamaterials, but that further analysis of a finite assembly should not be omitted. Even for relatively simple beam structures, a rich spectrum of spatial harmonics is present, and energy is converted from one wave type to another.

The results are important for practical applications of phononic crystals. Vibration isolation can only be achieved for high values of the imaginary wave number, which might be achieved in higher-order band gaps. It has to be taken into account that in partial band gaps, wave energy can be converted to other modes. Second, phononic crystals used for wave focusing or cloaking make use of the refractive index for a certain wave type. This property depends on the phase velocity, and therefore on the exact knowledge of the BZ in which the wave energy propagates. Our results show that a harmonic wave will be refracted at different angles, which implies that a single wave will be split into several beams with different wave speeds and wave numbers.
[1] P. A. Deymier, Acoustic metamaterials and phononic crystals, edited by P. A. Deymier (Springer, Heidelberg,2013).

[2] M. R. Haberman and M. D. Guild, Acoustic metamaterials, Phys. Today 69, 42-48 (2016).

[3] S. Zhang, L. Yin, and N. Fang, Focusing Ultrasound with an Acoustic Metamaterial Network, Phys. Rev. Lett. 102, 194301 (2009).

[4] P. Celli and S. Gonella, Manipulating waves with LEGO $^{\circledR}$ bricks: A versatile experimental platform for metamaterial architectures, Appl. Phys. Lett. 107, 081901 (2015).

[5] C. Zou, D. T. Lynd, and R. L Harne, Acoustic Wave Guiding by Reconfigurable Tessellated Arrays, Phys. Rev. Appl. 9, 014009 (2018).

[6] H. Chen, Y. Zhou, M. Zhou, L. Xu, and Q. H. Liu, Perfect Undetectable Acoustic Device from Fabry-Pérot Resonances, Phys. Rev. Appl. 9, 024014 (2018).

[7] A. Colombi, D. Colquitt, P. Roux, S. Guenneau, and R. V. Craster, A seismic metamaterial: The resonant metawedge, Sci. Rep. 6, 27717 (2016).

[8] V. Romero-García, J. V. Sánchez-Pérez, S. CastineiraIbánez, and L. M. Garcia-Raffi, Evidences of evanescent 
Bloch waves in phononic crystals, Appl. Phys. Lett. 96, 124102 (2010).

[9] C. Claeys, K. Vergote, P. Sas, and W. Desmet, On the potential of tuned resonators to obtain low-frequency vibrational stop bands in periodic panels, J. Sound Vib. 332, 1418-1436 (2013).

[10] V. Dorodnitsyn and B. Van Damme, Two-dimensional fluid-filled closed-cell cellular solid as an acoustic metamaterial with negative index, Phys. Rev. B 93, 134302 (2016).

[11] R. Fleury, A. B. Khanikaev, and A. Alù, Floquet topological insulators for sound, Nat. Commun. 7, 11744 (2016).

[12] Y. Chen, F. Qian, L. Zuo, F. Scarpa, and L. Wang, Broadband and multiband vibration mitigation in lattice metamaterials with sinusoidally-shaped ligaments, Extreme Mech. Lett. 17, 24-32 (2017).

[13] V. Laude, Y. Achaoui, S. Benchabane, and A. Khelif, Evanescent Bloch waves and the complex band structure of phononic crystals, Phys. Rev. B 80, 092301 (2009).

[14] Z. Liu, X. Zhang, Y. Mao, Y. Y. Zhu, Z. Yang, C. T. Chan, and P. Sheng, Locally resonant sonic materials, Science 289, 1734-1736 (2000).

[15] A. Bergamini, T. Delpero, L. De Simoni, L. Di Lillo, M. Ruzzene, and P. Ermanni, Phononic crystal with adaptive connectivity, Adv. Mater. 26, 1343-1347 (2014).

[16] D. M. Mead, Wave propagation in continuous periodic structures: Research contributions from Southampton, 1964-1995, J. Sound Vib. 190, 495-524 (1996).

[17] B. R. Mace and E. Manconi, Modelling wave propagation in two-dimensional structures using finite element analysis, J. Sound Vib. 318, 884-902 (2008).

[18] M. Rupin, F. Lemoult, G. Lerosey, and P. Roux, Experimental Demonstration of Ordered and Disordered Multiresonant Metamaterials for Lamb Waves, Phys. Rev. Lett. 112, 234301 (2014).

[19] F. Maurin, C. Claeys, E. Deckers, and W. Desmet, Probability that a band-gap extremum is located on the irreducible Brillouin-zone contour for the 17 different plane crystallographic lattices, Int. J. Solids Struct. 135, 26-36 (2017).

[20] H. Gersen, T. .J Karle, R. J. P. Engelen, W. Bogaerts, J. P. Korterik, N. F. Van Hulst, T. G. Krauss, and L. Kuipers, Direct Observation of Bloch Harmonics and Negative
Phase Velocity in Photonic Crystal Waveguides, Phys. Rev. Lett. 94, 123901 (2005).

[21] D. M. Profunser, O. B. Wright, and O. Matsuda, Imaging Ripples on Phononic Crystals Reveals Acoustic Band Structure and Bloch Harmonics, Phys. Rev. Lett. 97, 055502 (2006).

[22] F. Langfeldt, W. v. Gleine, and O. von Estorff, Analytical model for low-frequency transmission loss calculation of membranes loaded with arbitrarily shaped masses, J. Sound Vib. 349, 315-329 (2015).

[23] J. R . Willis, Negative refraction in a laminate, J. Mech. Phys. Solids 97, 10-18 (2016).

[24] L. Liu and M. I. Hussein, Wave motion in periodic flexural beams and characterization of the transition between Bragg scattering and local resonance, J. Appl. Mech. 79, 011003 (2012).

[25] D. Yu, J. Wen, H. Shen, Y. Xiao, and X. Wen, Propagation of flexural wave in periodic beam on elastic foundations, Phys. Lett. A 376, 626-630 (2012).

[26] M. I. Hussein, M. J. Leamy, and M. Ruzzene, Dynamics of phononic materials and structures: Historical origins, recent progress, and future outlook, Appl. Mech. Rev. 66, 040802 (2014).

[27] J. Wen, G. Wang, D. Yu, H. Zhao, and Y. Liu, Theoretical and experimental investigation of flexural wave propagation in straight beams with periodic structures: Application to a vibration isolation structure, J. Appl. Phys. 97, 114907 (2005).

[28] B. Van Damme and A. Zemp, Measuring dispersion curves for bending waves in beams: A comparison of spatial Fourier transform and inhomogeneous wave correlation, Acta. Acust. United Acust. 104, 228-234 (2018).

[29] J. Berthaut, M. N. Ichchou, and L. Jezequel, K-space identification of apparent structural behaviour, J. Sound Vib. 280, 1125-1131 (2005).

[30] L. Van Belle, C. Claeys, E. Deckers, and W. Desmet, On the impact of damping on the dispersion curves of a locally resonant metamaterial: Modelling and experimental validation, J. Sound Vib. 409, 1-23 (2017).

[31] R. P. Moiseyenko and V. Laude, Material loss influence on the complex band structure and group velocity in phononic crystals, Phys. Rev. B 83, 064301 (2011). 